\title{
Corela
}

Cognition, représentation, langage

14-2 | 2016

Vol. $14, \mathrm{n}^{\circ} 2$

\section{Affixes dérivationnels forts et faibles en agni et en baoulé}

\section{Alain Albert ADEKPATÉ}

\section{OpenEdition}

\section{Journals}

Édition électronique

URL : http://journals.openedition.org/corela/4619

DOI : $10.4000 /$ corela.4619

ISSN : $1638-573 \mathrm{X}$

\section{Éditeur}

Cercle linguistique du Centre et de I'Ouest - CerLICO

Référence électronique

Alain Albert ADEKPATÉ, « Affixes dérivationnels forts et faibles en agni et en baoulé », Corela [En ligne], 14-2 | 2016, mis en ligne le 12 janvier 2018, consulté le 19 avril 2019. URL : http:// journals.openedition.org/corela/4619; DOI : 10.4000/corela.4619

Ce document a été généré automatiquement le 19 avril 2019

\section{(c) (i) (2)(2)}

Corela - cognition, représentation, langage est mis à disposition selon les termes de la licence Creative Commons Attribution - Pas d'Utilisation Commerciale - Partage dans les Mêmes Conditions 4.0 International. 


\title{
Affixes dérivationnels forts et faibles en agni et en baoulé
}

\author{
Alain Albert ADEKPATÉ
}

\section{Introduction}

1 De nombreux travaux ont été consacrés à la dérivation qui soulignent son emploi comme l'un des procédés formels hautement productifs d'enrichissement lexical des langues (Wemers 1973 ; Guilbert 1975 ; Matthew 1991 ; Katamba 1993). La dérivation s'opère par le biais d'un certain nombre de moyens formels qui sont la variation de schème tonal (DikiKidiri 1977 : 72-74, 2008 : 228-229), le redoublement tonal ou partiel de l'unité de base, et l'adjonction d'affixes, c'est-à-dire la préfixation, la suffixation, la parasynthèse ou circonfixation, l'infixation et l'interfixation (Sow 1966 et Nwankwegu 2013).

2 Le baoulé et l'agni, deux langues classées comme appartenant à la branche tano centrale de la famille kwa, attestent des faits de dérivation par affixation à une base verbale. Pour un même affixe, la dérivation tantôt n'entraîne aucun effet perceptible sur le schème tonal, tantôt n'affecte que celui de la base verbale ou bien celui de la base verbale et de l'affixe, à la fois. Cela peut être illustré en baoulé (du moins en Agba, un dialecte baoulé) par l'adjonction à des bases verbales des suffixes -fwè et -wâ qui permettent de dériver

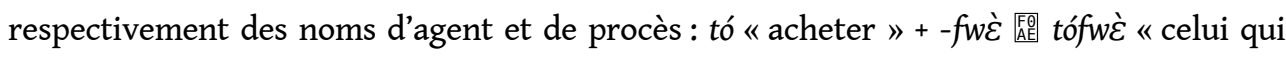
achète/client »/ $s r \check{\varepsilon}$ «demander, faire une requête »+-fwì 原 $s r \grave{\varepsilon} f w \hat{\varepsilon}$ 《demandeur/

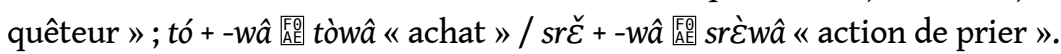

Des faits similaires s'observent en agni et ils posent l'épineux problème du fonctionnement tonal des affixes de dérivation et des bases verbales dans les formations dérivées de ces langues.

4 L'hypothèse qui sous-tend notre approche est que dans ces langues, et peut-être dans la plupart des langues tano, regroupant aussi le krobou, l'abouré, l'éotilé, l'akan, le nzémaahanta, l'anuf,, l'efutu-awutu, le larteh-cherepong-anum et le guang nord (Stewart J. 1989 cité par Blench R. et W. Kay 2000 : 29), les affixes dérivationnels se répartissent en 
deux classes majeures: celle des affixes forts et celle des affixes faibles. L'adjonction d'affixes forts va déclencher un processus tonal systématique obligatoire d'abaissement $\mathrm{du}$ ton du verbe, au premier niveau, le facteur dialectal pouvant permettre que l'affixe faible, qui est en principe dépourvu d'un tel pouvoir, ait un effet abaisseur du ton de la base verbale. A l'inverse, à un deuxième niveau, du fait du facteur dialectal, un autre processus tonal orienté vers le suffixe peut être déclenché. Ces processus ont pour effet la formation soit d'un domaine morpho-phonologique constitué par la forme dérivée : [base verbale + affixe], au premier ou au deuxième niveau, soit de deux domaines morphophonologiques constitués l'un de la base et l'autre de l'affixe : [base verbale][affixe].

Quelle est la nature des processus tonals en jeu dans la dérivation des noms à partir des bases verbales? Avec quels affixes et de quelle(s) façon(s) opèrent-ils ? A ces questions, nous essaierons d'apporter des réponses dans la perspective de l'interface morphologiephonologie. Dans le premier point, nous présentons les données illustrant les faits d'affixation en question en baoulé et en agni. Le deuxième point est consacré aux analyse et discussion des faits.

\section{Présentation des données}

Les données à l'étude ont été collectées auprès de locuteurs natifs du baoulé et de l'agni. Ci-dessous, nous les présentons selon le type d'affixe, c'est-à-dire selon qu'il s'agit de préfixes, de suffixes ou de circonfixes (préfixe...suffixe), et selon la structure syllabique de la base verbale et par conséquent le schème tonal qui lui est associé.

7 Il faut indiquer que pour le baoulé, nous avons recueilli les données auprès de locuteurs natifs de trois parlers ${ }^{1}$ : celui de Toumodi, considéré comme le parler central (Cent), le gblo (Gb) qui est parlé dans la sous-préfecture de Diabo et l'agba (Agb), parlé dans la souspréfecture de Bocanda. Pour l'agni, nous avons retenu trois parlers ${ }^{2}$, le sanvi (san), parlé principalement à Aboisso, l'ànó (An), parlé à Prikro et l'indénié (Ind), qui est parlé à Abengourou.

\subsection{Faits de dérivation affixale dans les parlers baoulé}

$8 \quad$ Les données recueillies dans les parlers baoulé concernent un certain nombre d'affixes qui sont soit identiques soit très peu variables d'un parler à un autre, avec la même valeur, et qui sont combinables à des base verbales auxquelles sont associés différents schèmes tonals que Creissels et Kouadio (1977: 373-394) désignent comme 'ton fondamental', c'est-à-dire un schème tonal (phonologique) qui dépend de la structure syllabique de ces bases : ce sont cv́, cčv, cv̀v et c̀vcv́. Les parlers n'admettent pas tous les mêmes affixes.

- Suffixes:

i) -fwè (Agb) et -fwê (Cent, Gb) combinés à des bases verbales de type cv́, ccv̌, c̀vv́ et (c)v̀cv́ pour dériver des noms d'agent ou signifiant « celui qui fait ou est impliqué dans l'action de » :

\begin{tabular}{|c|c|c|}
\hline Verbe + -fwè (Agb) & Verbe $+-f w \hat{\varepsilon}(G b)$ & Verbe $+-f w \hat{\varepsilon}$ (Cent) \\
\hline
\end{tabular}




\begin{tabular}{|c|c|c|c|}
\hline (1) & a. tó « acheter » $\rightarrow$ tófwè « client » & a'. tófw $\hat{\varepsilon} \ll$ id. » & a". tófwêع «id.» \\
\hline & b. srì « rire » $\rightarrow$ srifw $\hat{\varepsilon}$ « moqueur » & b'. srifwêع «id. » & b". srifwê «id.» \\
\hline & c. (j)ìjó « parler » $\rightarrow(j)$ iłófwè « locuteur » & $c^{\prime} .(j)$ ijòfw $\hat{\varepsilon}$ «id. » & c". jiłófwê «id. » \\
\hline & d. tié « écouter » $\rightarrow$ tjèfw $\hat{\varepsilon}$ « celui qui écoute & d'. tjèfwê «id. » & d". tjèfwêع «id.» \\
\hline
\end{tabular}

9 ii) -lर्ع (Cent, Ag, Gb) combinés à des bases verbales de type cv́, ccv̌ et cv̀cv́ pour former des noms de procès ou désignant le résultat d'une action. Les affixes en (2a.-c.) sont admis dans le parler central et le gblo:

\begin{tabular}{|c|c|c|}
\hline & Verbe $+-1 \hat{\varepsilon}(A g b)$ & 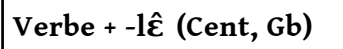 \\
\hline \multirow[t]{4}{*}{ (2) } & a. kó « partir » $\rightarrow$ kólî « départ » & a'. kólêع «id.» \\
\hline & b. srǐ « rire » $\rightarrow$ srìl $\hat{\varepsilon}$ « moquerie » & b'. srillê «id. » \\
\hline & 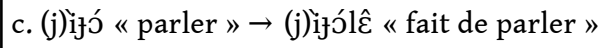 & $c^{\prime} .(j)$ ijòl $\hat{\varepsilon}$ « id. » \\
\hline & d. tié « écouter » $\rightarrow$ tjèl $\hat{\varepsilon}$ « fait d'écouter » & d'. tjèlêع « fait d'écouter » \\
\hline
\end{tabular}

iii) -wâ (Agb) ou -wá (Gb) + verbes cv́, ccv̌ et cv̀cv́ :

\begin{tabular}{|l|l|l|}
\hline & Verbe + -wâ (Agb) & Verbe + -wá (Gb) \\
\hline$(3)$ & a. kó « partir » $\rightarrow$ kòwâ « départ » & a'. kòwá « id. » \\
\hline & b. klě « écrire » $\rightarrow$ klèwâ « écriture » & b'. klèwá « id. » \\
\hline & c. (j)ìjó « parler » $\rightarrow$ (j)ìjòwâ « bavardage » & c'. (j)ìjòwá « id. » \\
\hline
\end{tabular}

11 iv) -wlर (Cent, Gb) + verbes cv́, cč̌, cvvv́ et cv̀cv́ :

\begin{tabular}{|c|c|}
\hline & Verbe + -wlê (Cent, Gb) \\
\hline \multirow[t]{4}{*}{ (4) } & a. lá « se coucher » $\rightarrow$ làwl $\hat{\varepsilon}$ « logement » \\
\hline & b. fiá « se cacher » $\rightarrow$ fiàwlêع « cachette » \\
\hline & c. tră « habiter » $\rightarrow$ trà̀wl $\hat{\varepsilon}$ 《 habitation » \\
\hline & d. sìké « décharger » $\rightarrow$ sikèwlê « lieu où l'on dépose la charge pour souffler un peu » \\
\hline
\end{tabular}

v) -ljê (Agb, Gb) et -ljě (Cent) combinés à des bases verbales de type cv́ et cv̀cv́ pour former des noms d'instruments ou de pratiques : 


\begin{tabular}{|c|c|c|}
\hline & Verbe $+-\operatorname{lj} \hat{\varepsilon}(A g b, G b)$ & Verbe $+-\operatorname{lj} \check{\varepsilon}$ (Cent) \\
\hline \multirow[t]{2}{*}{ (5) } & a. kpé « couper » $\rightarrow$ kpèljế « hâche » & a’. kpèljě « id. » \\
\hline & b. kpàkpá « frotter » $\rightarrow$ kpàkpàljêع « pommade » & b. kpàkpàljě «id." \\
\hline
\end{tabular}

13 À la différence phonique près, ći « interdire » des parlers central et agba se dit kí en gblo. Les effets de la dérivation par -ljê et -ljž sur les schèmes tonals sont les mêmes dans tous les parlers.

\section{- Préfixes :}

i) à- (Cent, Agb, Gb) se préfixe à des bases verbales de type cv́, cvv́ pour former des noms de procès ou désignant le résultat d'une action :

\begin{tabular}{|l|l|l|}
\hline & à- + Verbe & \\
\hline (6) & a. f́́ « se fatiguer » $\rightarrow$ àf̌̌ « fatigue » & b. jiá « rassember » $\rightarrow$ àjă « assemblée » \\
\hline
\end{tabular}

ii) $\mathrm{N}-(\mathrm{Cent}, \mathrm{Agb}, \mathrm{Gb})$ se combine à des bases verbales de type c̀vv́ et cv̀cv́ pour former des noms d'entité ou de procès se rapportant au procès désigné par le verbe :

\begin{tabular}{|l|l|}
\hline & ̀̀ + Verbe \\
\hline (7) & a. bié « uriner » $\rightarrow$ m̀mìe « urine » \\
\hline & b. sié « garder, enterrer » $\rightarrow$ ǹzì́ « don fait lors de funérailles » \\
\hline & c. kpàtá « supplier, demander pardon » $\rightarrow$ ỳgbàtá « pardon » \\
\hline
\end{tabular}

\section{- Circonfixes :}

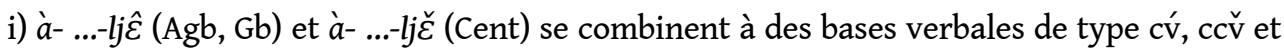
cvvv pour former des noms de lieux (sens dénotatif ou connotatif), de propriété ou désignant une façon de faire ou d'être :

\begin{tabular}{|c|c|c|}
\hline & à-+ Verbe+-ljê $(A g b, G b)$ & à-+ Verbe+-ljě (Cent) \\
\hline \multirow[t]{3}{*}{ (8) } & a. sié $\rightarrow$ àsièljî « cimetière » & a'. àsì̀ljě « id. » \\
\hline & 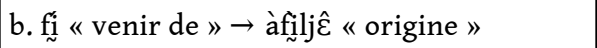 & b'. àfiljě «id. » \\
\hline & c. gwă « verser » $\rightarrow$ àgwàljê « destination » & c'. àgwàljě « id.» \\
\hline
\end{tabular}


ii) $\grave{N}-$...-ljê (Agb, Gb) et $\grave{N}-$...-ljě (Cent) se combinent à une base verbale de type cv́, ccv̌ et c̀v́ pour former des noms de lieux ou désignant le résultat du procès exprimé par le verbe :

\begin{tabular}{|c|c|c|}
\hline & $\grave{\mathbf{N}}-+$ Verbe + -ljê(Agb, Gb) & $\grave{N}-+$ Verbe +-ljě (Cent) \\
\hline \multirow[t]{3}{*}{ (9) } & a. kpá « coudre » $\rightarrow$ ỳgbàljê « couture » & a'. ̀̀gbàljě «id. » \\
\hline & b. klě « écrire » $\rightarrow$ ỳglèljê « écriture » & b'. ỳglèljě «id. » \\
\hline & c. fiá « se cacher » $\rightarrow$ m̀viàljês « affût » & c'. m̀viàljě «id. » \\
\hline
\end{tabular}

\subsection{Faits de dérivation affixale dans les parlers agni}

Pour l'agni, nos données ne concernent que les verbes de structures cv́, cviv et cv̀cv́. Les verbes cč̌ ne sont pas retenus, en raison du fait que, comme le suggèrent ou l'attestent des travaux sur l'agni (A. Adou 2015 : 108, 128-129 ; A. Assanvo 2009 : 41, 50-51 et M. Kéita 2008), les formes et particulièrement les formes verbales de structures $c_{1} c_{2} \breve{v}$, où - $c_{2}-=1 / \Omega$, sont en variation libre avec celles de structure $c_{1} \grave{v}_{1} c_{2} v_{2}$, la structure $c_{1} c_{2} \check{v}$ étant interprétée comme résultant de la syncope de $-\mathrm{v}_{1}$ - et la réassociation du ton de cette dernière à $\mathrm{v}_{2}$.

L'ànó a, à l'exception de $\grave{N}-$...-ljě (aussi noté $\grave{N}-$...-lìé ), l'équivalent ou le quasi-équivalent de tous les affixes attestés en baoulé. L'indénié en atteste un peu moins, et apparemment

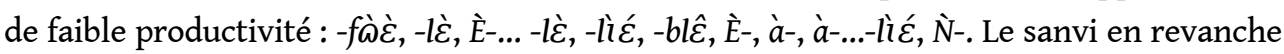

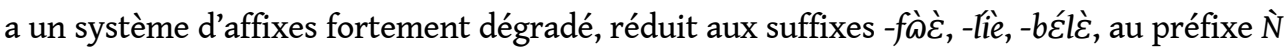

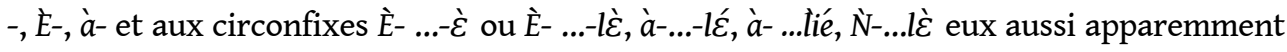
de faible productivité.

i) -fò غ̀ (An, San, Ind) se combine à une base verbale de type cv́, cvvv́ ou cv̀cv́ pour former des noms à valeur agentive ou signifiant " celui qui fait ou est impliqué dans l'action de ». En (10) ci-dessous, (a.)-(a".) illustrent les faits tels qu'ils se manifestent en ànó, (b.)-(b”.) ceux de l'indénié et (c.)-(c".) ceux du sanvi :

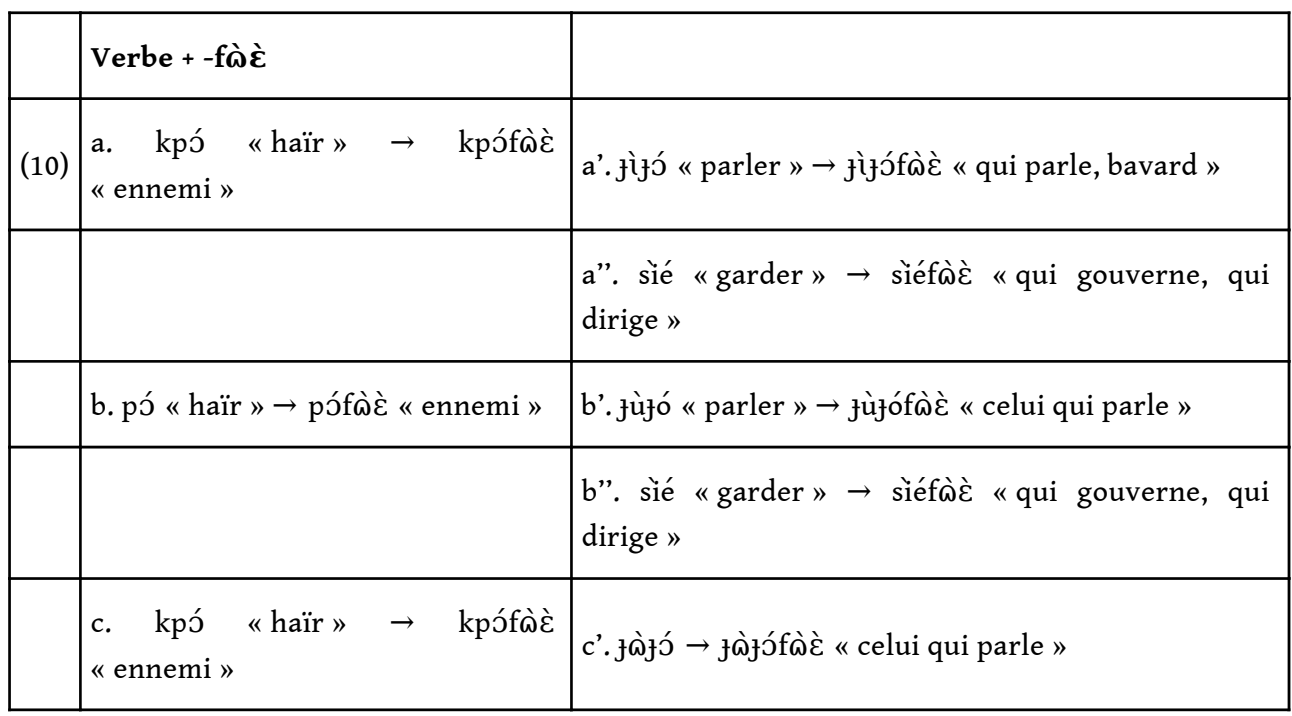




\begin{tabular}{|l|l|}
\hline & $\begin{array}{l}\text { c". sié «garder » } \rightarrow \text { siéfò } \varepsilon \text { « qui gouverne, qui } \\
\text { dirige » }\end{array}$ \\
\hline
\end{tabular}

19 ii) -lغ̀ est attesté en indénié et en ànó. Il se suffixe à des verbes de type cv́, c̀vv́ et cv̀cv́ pour dériver des noms de procès.

\begin{tabular}{|c|c|c|}
\hline & Verbe + -lغ̀ (An) & Verbe + -lè (Ind) \\
\hline \multirow[t]{6}{*}{ (11) } & a. kó « partir» $\rightarrow$ kólè « départ » & a'. kólè « id.» \\
\hline & b. fí « vomir » $\rightarrow$ fílè « le fait de vomir » & b'. fílè «id.» \\
\hline & 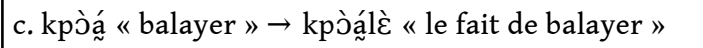 & c’. pìa làlè « id.» (vb. pìa ) \\
\hline & d. sié " garder, diriger » $\rightarrow$ siélè « le fait de diriger » & d'. siélè « id. » \\
\hline & 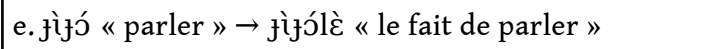 & e'. jùjólè «id. » (vb. jùjó) \\
\hline & f. nà̀tí « marcher » $\rightarrow$ nà̀tílè « le fait de marcher » & f'. nà̀dílè «id.» (vb. nà̀dí) \\
\hline
\end{tabular}

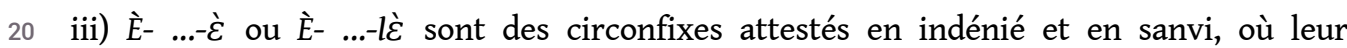
affixation à des verbes de type cv́, cv̀v et cv̀cv́ donne souvent lieu non seulement au phénomène d'harmonie de trait ATR mais aussi à des faits de variation formelle dont le plus caractéristique est la possibilité pour l'élément suffixal de ne pas se manifester segmentalement dans la forme dérivée.

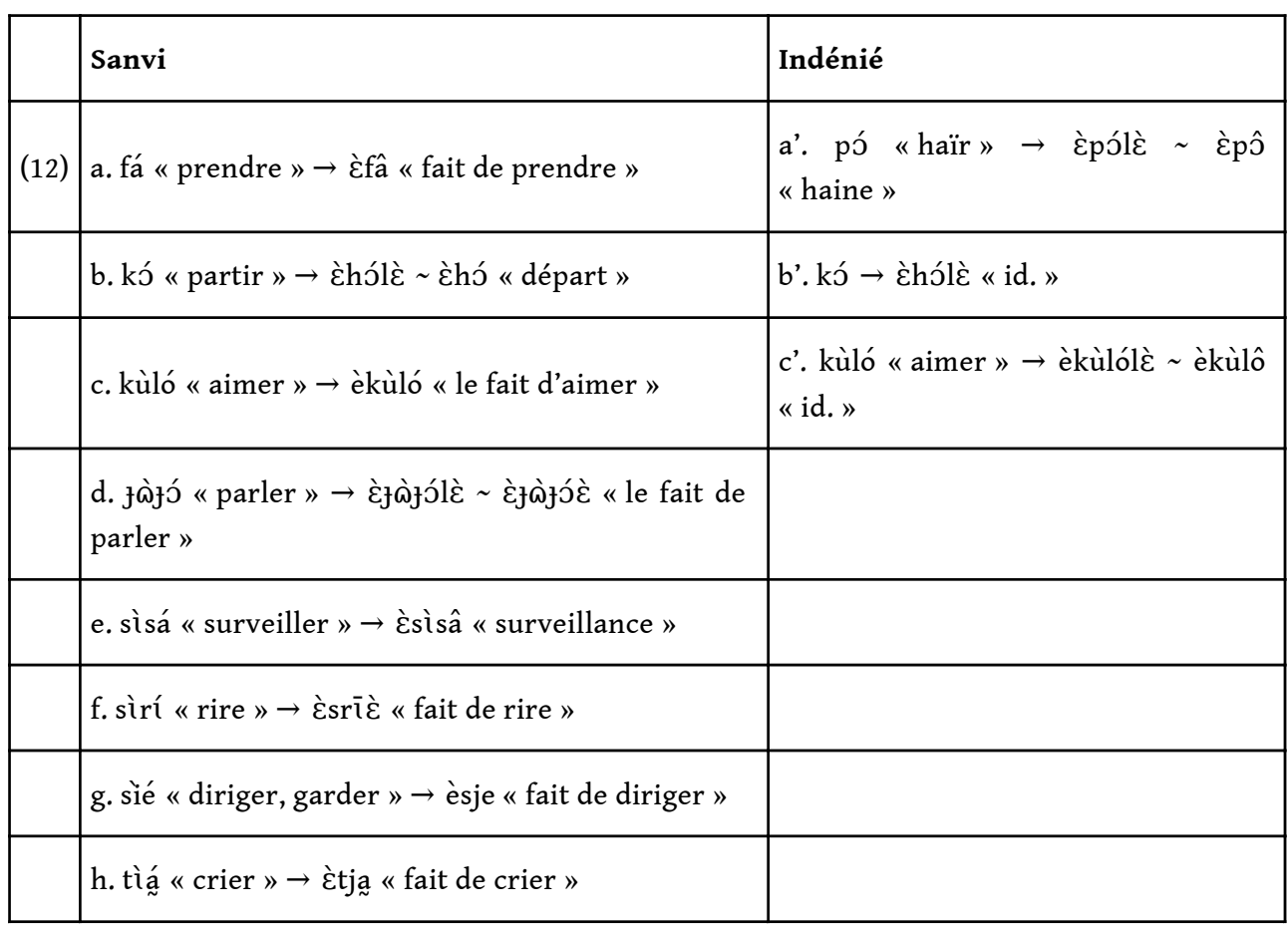

iv) Ǹ-...-lغ̀ est un circonfixe dont l'emploi est attesté avec quelques rares verbes en sanvi. 
(13) dàfí « dormir » $\rightarrow$ ǹnàfílè « sommeil »

v) ̀- est un préfixe attesté en ànó (a-d), en indénié (ex. a'-d') et en sanvi (ex. a"-c") avec des verbes de structures cv̀v́ et cv̀cv́.

\begin{tabular}{|c|c|c|c|}
\hline (14) & a. bié « uriner » $\rightarrow$ m̀mié « urine » & a'. m̀mié « id. » & $\begin{array}{l}\text { a". mimié } \\
\text { «id.» }\end{array}$ \\
\hline & $\begin{array}{l}\text { b. sié "garder» } \rightarrow \text { ǹzié «don fait lors de } \\
\text { funérailles" }\end{array}$ & b'. ǹziêe «id. » & \\
\hline & $\begin{array}{l}\text { c. kpàtá " demander pardon " } \rightarrow \text { ỳgbàtâ } \\
\text { «pardon » }\end{array}$ & $\begin{array}{l}\text { c'. m̀̀bàtâ «id. » (vb. } \\
\text { pàtá) }\end{array}$ & $\begin{array}{l}\text { c". ỳgbàtá } \\
\text { «id.» }\end{array}$ \\
\hline & 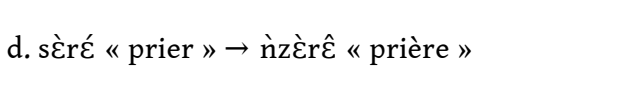 & $\begin{array}{l}\text { d'. kpà̀zá «se } \\
\text { promener » }\end{array}$ & \\
\hline & & $\begin{array}{l}\rightarrow \text { ỳgbà̀zâ } \\
\text { «promenade » }\end{array}$ & \\
\hline
\end{tabular}

vi) $\grave{E}$ - / ’a-sont des préfixes apparemment d'emploi limité et de distribution inégale dans les parlers agni, aptes à se combiner à des verbes de type cv́ et c̀vv́ pour dériver aussi bien des noms de procès que des noms d'agent. Cela peut être illustré par les exemples ciaprès, en ànó ( $a-b)$, en indénié (ex. a'-b') et en sanvi (ex. a"-b")

\begin{tabular}{|c|c|c|c|}
\hline (15) & 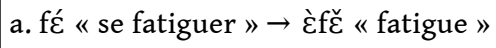 & a'. غ̀f̌̌ « id. » & a". غ̀f $\tilde{\varepsilon}$ «id.» \\
\hline & b. łá « épouser » $\rightarrow$ àjă « mariage » & b'. àjă «id. » & b". àjă «id. » \\
\hline
\end{tabular}

La nature du préfixe et la structure de la base verbale suggèrent que l'on classe parmi ces formes nominales dérivées en (15) celle que nous avons en (16) ci-dessous :

\begin{tabular}{|l|l|l|l|}
\hline$(16)$ & a. tá « élever » $\rightarrow$ c̀tâ « fait d'être nourrice » & a'. c̀tâ «id. » & a". c̀tâ « id. » \\
\hline
\end{tabular}

vii) -lić, attesté en ànó et en indénié, et -liee, en sanvi, sont des suffixes apparemment aussi d'emploi très limité qui se suffixent à un verbe de type cv́ pour former des noms d'instrument, de procès.

\begin{tabular}{|c|c|c|c|}
\hline (17) & a. cí « interdire » $\rightarrow$ cilìć « totem » & a'. cillìć « totem » & a". cilíè « totem » \\
\hline
\end{tabular}


viii) $a$-...-lì é est attesté en ànó et en indénié, $a$ a-...-lć en sanvi, où il est d'emploi très rare. Ces morphèmes s'affixent à des verbes de type cv́ et cv̀v pour dériver des noms de lieux et de procès.

\begin{tabular}{|c|c|c|c|}
\hline (18) & a. wó « accoucher » $\rightarrow$ àw@̀lì $\varepsilon$ « accouchement » & a'. àwòllé « id.» & a". àwòlć «id.» \\
\hline & b. bìá « se laver » $\rightarrow$ àbìàlić « douchière » & b'. àbiàlì ع́ «id.» & b". àbiàlé «id.» \\
\hline & c. sié « garder » $\rightarrow$ sièlì $\varepsilon$ « cimetière » & c’. àsièlìć «id. » & \\
\hline
\end{tabular}

ix) -wâ est attesté seulement en ànó, où il se suffixe à verbes de type cv́, c̀vv́ et cv̀cv́ pour dériver des noms de procès.

\begin{tabular}{|c|c|}
\hline \multirow[t]{2}{*}{ (19) } & a. kó « partir » $\rightarrow$ kòwâ « départ » \\
\hline & 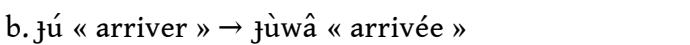 \\
\hline & c. sié « garder » $\rightarrow$ sìèwâ « fait de garder » \\
\hline & 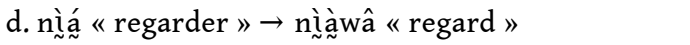 \\
\hline & e. nì̀kí « chatouiller » $\rightarrow$ nì kìwwâ « chatouilleme \\
\hline
\end{tabular}

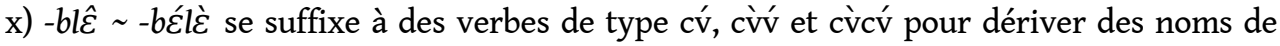
lieux ou ayant comme signification «occasion de ». En sanvi, où il est de fréquence d'emploi très rare, on le retrouve sous la forme -bćlè.

\begin{tabular}{|c|c|c|c|}
\hline (20) & a. dá « se coucher » $\rightarrow$ dàblî « couchette » & a'. dàblêع «id.» & a". dàbćlè «id.» \\
\hline & b. fiá « se cacher » $\rightarrow$ fiàblêع « cachette » & b’. fiàblêع «id.» & \\
\hline & 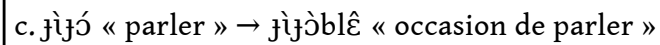 & c'. jùjòblêع «id. » & \\
\hline
\end{tabular}

\section{Analyse et discussion}

L'adjonction des affixes aux bases verbales laisse apparaître un certain nombre de régularités de fonctionnement tonal dont il nous revient de rendre compte et de proposer une interprétation.

\subsection{A propos des notions d' 'affixe fort' et d' 'affixe faible'}

Un parcours minutieux des faits montre que dans les formes dérivées, les suffixes -fwì /$f w \hat{\varepsilon},-l \hat{\varepsilon}$, le préfixe $\grave{N}-$ du baoulé d'une part, et les suffixes -fò̀, $-l \grave{\varepsilon}$, le préfixe $\grave{N}^{-}$et les circonfixes $\grave{E}-\ldots-. \bar{\varepsilon}$ ou $\grave{E}-\ldots-.-l \grave{\varepsilon}, \grave{N}-\ldots-.-l \grave{\varepsilon}$ de l'agni, d'autre part, n'ont globalement pas d'effet dépresseur sur le ton fondamental de la base verbale, puisque celui-ci se conserve, même 
si des faits de combinatoire, dont nous rendrons compte plus loin, font qu'en surface, la reconnaissance du ton fondamental est un peu brouillée. En revanche, les suffixes -ljê /-

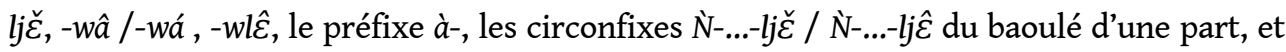

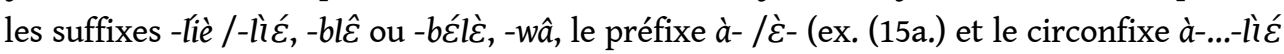
/ à- ...-lé, ont un effet dépresseur notable sur le ton de la base verbale, car ils favorisent un abaissement du ton fondamental du verbe qui devient Bas (B) pour les verbes de structure cv́ et cč̀, Bas-Bas (B-B) pour les verbes de structure c̀vv́ et cv̀cv́. L'effet abaisseur de ces affixes dérivationnels peut être assimilé à celui que produisent les expansions ou circonstants postposés aux verbes, au niveau phonologique en baoulé (Creissels \& Kouadio 1977 : 377-394). Cet effet consiste à abaisser le « demarcative high tone » du verbe qui « automatically attaches to its last vowel whenever it is not followed by a complement or adjunct. $\rrbracket^{3}$ (Creissels \& Kouadio (2010: 167). Un fait similaire a été identifié en agni indénié par Leben (1981:178) et en ànó par Adou (2015), puis confirmé par l'ensemble de nos informateurs. Ce que les faits ci-dessus présentés suggèrent, c'est que les affixes qui se caractérisent par l'absence d'effet sur les verbes doivent être interprétés comme des affixes faibles et ceux qui ont un effet abaisseur sur les tons verbaux comme des affixes forts.

\subsection{Mécanismes tonals impliqués dans la dérivation et problèmes}

31 Si l'essai de généralisation ci-dessus établit la dichotomie entre les affixes que nous avons convenu de désigner comme 'forts' du fait de leur effet abaisseur sur les tons des verbes et les affixes 'faibles' qui ne produisent pas un tel effet, il laisse cependant subsister certaines questions qui sont de plusieurs ordres et qui méritent d'être examinées :

- qu'est-ce qui explique qu'en apparence, en baoulé, les verbes de structures cv̀cv́ du gblo (cf. ex. (1c'.)) et cč̌ des parlers gblo et central (cf. ex. (1b'.) et (1b”.)) subissent un effet d'abaissement tonal au contact du suffixe -fwÊ , en dépit du caractère faible postulé pour celui-ci ? Pourquoi avec le même suffixe, pour l'ensemble des parlers, la structure c̀vv́ du verbe devient-elle ccv̌ et pourquoi le suffixe agba -fwè devient-il - fwế dans un tel contexte (cf. ex. (1d.), (1d'.) et (1d".)) ?

- qu'est-ce qui explique que le ton des verbes de structure cv̀cv́ des parlers gblo et central (cf. (2c'.)) et ceux de structure ccv̌ de l'ensemble des parlers (cf. ex (2b.) et (2b'.)) s'abaissent au contact du suffixe $-1 \hat{\varepsilon}$ ? Comment expliquer que pour l'ensemble des parlers, les verbes de structure cvv́ se réalisent ccv̌ au contact de ce suffixe (cf. ex. (2b.), $\left.\left(2 b^{\prime}.\right) /(2 d),.\left(2 d^{\prime}.\right)\right)$ ?

34 En agni, les cas problématiques restent ceux qui découlent particulièrement de

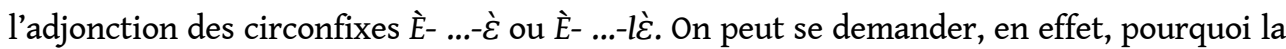
position de l'élément suffixal des circonfixes n'est pas avec certains verbes segmentalement pourvue (cf. ex. (12a.), (12a'.), (12c.), 12c'.), (12e.)-(12h.)), pourquoi la structure cv̀rv́ des verbes devient crv̄, et pourquoi cvvv́ devient cjv̄ dans la forme dérivée (cf. ex. (12f.)-(12h.)).

Pourquoi, avec le préfixe faible $\grave{N}-$, y a-t-il une modulation sur la syllabe finale de certaines formes dérivées (cf. ex. (14b'.), (14c.), (14c'), (14d.) et (14d')) ? L'autre problème épineux à résoudre, c'est celui de la détermination du statut du préfixe $\grave{E}$ - qui, comme à-, a un effet dépresseur sur le ton du verbe, ainsi que cela est illustré en (15), tout comme d'ailleurs le préfixe à- attesté en baoulé, mais qui, en (16), ne semble pas avoir cet effet. 
Il y a d'autres faits, bien communs parmi les langues kwa. Le premier concerne les processus phonologiques déclenchés par la préfixation de la nasale syllabique $\mathrm{N}-$. Préfixée, cette consonne déclenche un processus phonologique d'homorganisme, se réalisant selon le trait de position de la consonne qu'elle précède en même temps qu'elle la sonorise si elle est sourde ou la nasalise si elle est orale. Le second est celui de l'harmonie vocalique selon le trait ATR. On peut en voir la manifestation entre l'élément préfixal $\grave{E}$ - des circonfixes et les voyelles de la base verbale, le premier se réalisant $\grave{\varepsilon}$ lorsqu'il est préfixé à une base verbale à voyelle -ATR (cf. ex. (12.a)-(12a'), (12b)-(12b'), (12d)-(12d'), (12e)-(12e') / (12c)-(12c'), (12g)). Ce sont là des faits qui relèvent purement du niveau segmental et de moindre implication pour le traitement de nos données.

Nous pouvons à présent définir le cadre général de nos analyses des faits en baoulé et en agni. Ce cadre d'analyse présente deux niveaux: le niveau I où peuvent intervenir des règles tonales assorties de conditions, impliquant les suffixes forts ou faibles, dans la directionalité droite-gauche ou bien des préfixes forts ou faibles, dans la directionalité gauche droite. Les affixes faibles ne produisent en principe aucun effet d'abaissement tonal mais leur adjonction peut déclencher l'application de règles qui créent les conditions du passage au niveau II, où vont s'appliquer d'autres règles, de directionalité gauche-droite, pour produire les formes dérivées attendues.

Au niveau I, peuvent s'appliquer les règles suivantes :

- (R I) : Règle de resyllabification qui s'applique à la base verbale de structure $\mathrm{cv}_{1} \mathrm{v}_{2}$ et qui consiste en plusieurs opérations que nous énonçons dans les sous-règles suivantes :

- (R Ia) : propagation $\mathrm{T}_{1}$ (Ton) sur la position de $\mathrm{v}_{2}$ qui s'applique avec les verbes de structure $\mathrm{Cv}_{1}(\mathrm{c}) \mathrm{v}_{2}$;

- (R Ib) : Règle de désassociation de $\mathrm{T}_{1}$ qui s'applique au ton de $\mathrm{v}_{1}$;

- (R Ic) : Règle de palatalisation qui s'applique à $\mathrm{v}_{1}$ sans ton après désassociation de $\mathrm{T}_{1}$. La condition est que $\mathrm{v}_{1}$ est antérieure et $+\mathrm{H}\left(\mathrm{v}_{1}\right.$ plus haute) ;

- (R Id) : Désassociation de $\mathrm{T}_{2}$ du verbe qui s'applique aux verbes dont la structure $\mathrm{c}_{1} \mathrm{c}_{2} \mathrm{v}$ est obtenue après la resyllabification ;

- (R Ie) : Effacement de T flottant (noté entre parenthèses). La condition ici est que T flottant $=\mathrm{T}$ suivant. Cette règle peut s'appliquer indépendamment des précédentes, c'està-dire en dehors de tout processus de resyllabification.

39 Au niveau I, il y a aussi d'autres règles qui permettent de dériver des formes, sans avoir recours à la resyllabification. Il s'agit en particulier de :

- (R I') : Règle d'abaissement automatique du Ton Haut démarcatif.

Au niveau II, peut intervenir une règle d'association du ton flottant (R II). La condition ici est que $\mathrm{T}$ flottant $\neq \mathrm{T}$ suivant non-flottant. Son application est de directionalité gauchedroite. 
41 Les domaines morpho-phonologiques sont indiqués entre crochets. Et la frontière morpho-phonologique à un certain niveau est indiquée par une ligne verticale en gras entre les domaines. Par exemple : [Verbe] 原 $[$ [affixe].

Dans un souci d'atteindre un niveau raisonnable de généralité, les verbes sont représentés par leur structure syllabique.

43 Pour la commodité de la description et de l'analyse des faits et comme nous le suggèrent nos données de l'agni, nous ne prendrons en compte que l'élément suffixal des circonfixes, dans leur relation à la base verbale. C'est en effet cet élément qui est affecté dans la forme lorsque le circonfixe s'adjoint à une base verbale.

\section{a. Le cas du baoulé}

44 Ci-dessous, nous analysons, au regard de ce qui précède, les faits justifiant de reconnaître des affixes du baoulé comme 'faibles' ou comme 'forts'.

45 - Les affixes faibles

Nous avons vu que dans cette langue, il y a trois affixes faibles $-f w \grave{\varepsilon} /-f w \hat{\varepsilon},-l \hat{\varepsilon}$ et $\grave{N}-$.

Cependant, dans la pratique, les deux suffixes $-f w \hat{\varepsilon}$ et $-l \hat{\varepsilon}$ ont des emplois où ils déclenchent l'effet d'abaissement tonal reconnu aux suffixes forts. Ce sont des emplois conditionnés par les différences d'ordre dialectal mais aussi apparemment d'ordre phonologique, si on se fie à la structure syllabique des verbes concernés. En effet, le suffixe -fw $\hat{\varepsilon}$ se rencontre, dans un tel emploi, dans le parler gblo, après un verbe de structure $c_{1} v_{1} c_{2} v_{2}$. Le suffixe $-l \hat{\varepsilon}$, dans un tel contexte d'emploi, se rencontre dans les parlers central et gblo. Nous ne nous prononcerons pas plus sur la question mais nous nous limiterons ici, dans l'attente de recueillir davantage de données par le recours autres dialectes du baoulé, à postuler que les affixes en question sont faibles.

- Avec les verbes de structure cv́ : 
$(21)$
a. cv́-fwè

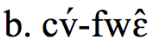

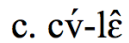

\section{Niveau I}

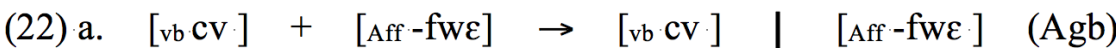

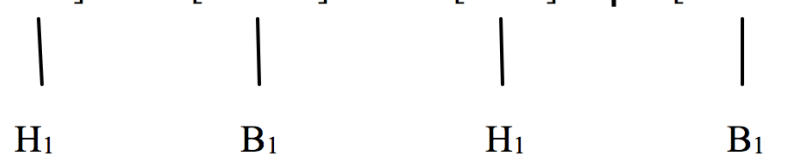

b. $\quad[\mathrm{vb} \cdot \mathrm{cv}]+[\mathrm{Aff}-\mathrm{fw} \varepsilon] \rightarrow[\mathrm{vb} \cdot \mathrm{cv}] \mid[\mathrm{Aff}-\mathrm{fw} \varepsilon] \quad(\mathrm{Gb}, \mathrm{Cent})$

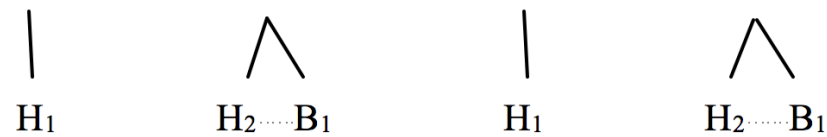

c. $[\mathrm{vb} \mathrm{Cv}]+[\mathrm{Aff}-1 \varepsilon] \rightarrow[\mathrm{vb} \mathrm{Cv}] \mid[\mathrm{Aff}-1 \varepsilon](\mathrm{Agb}, \mathrm{Gb}, \mathrm{Cent})$

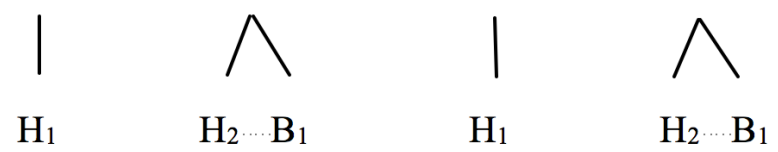

L'adjonction des affixes n'entraîne aucune variation du ton verbal. La dérivation de ces formes a lieu au niveau I, avec la formation de deux domaines morpho-phonologiques.

- Avec les verbes de structure ccv̌ :
(23) a. $c_{1} c_{2} \grave{v}-f w \hat{\varepsilon}(A g b)$
b. $c_{1} c_{2} \grave{v}-f w \hat{\varepsilon}$ ( Cent, Gb)
c. $\mathrm{c}_{1} \mathrm{c}_{2} \grave{\mathrm{v}}-\mathrm{l} \hat{\varepsilon}(\mathrm{Agb}, \mathrm{Gb}, \mathrm{Cent})$

\section{Niveau I}

(24) $\left.\left[\mathrm{vb}_{1} \mathrm{C}_{2} \mathrm{~V}\right]+[\mathrm{Aff}-\mathrm{fw} \varepsilon] \rightarrow *\left[\mathrm{vb}_{1} \mathrm{c}_{1} \mathrm{C}_{2} \mathrm{~V}\right] \mid{ }_{\mathrm{Aff}}-\mathrm{fw} \varepsilon\right]$ (Agb)

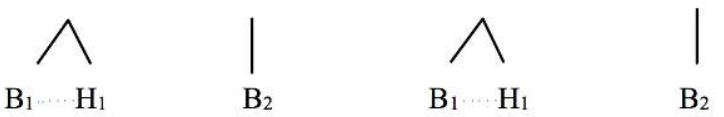

La structure obtenue au terme du processus en (24) n'est pas la forme attendue de (23a). Cette structure se prête par conséquent à l'application de la règle ( $\mathrm{R} \mathrm{Id).}$

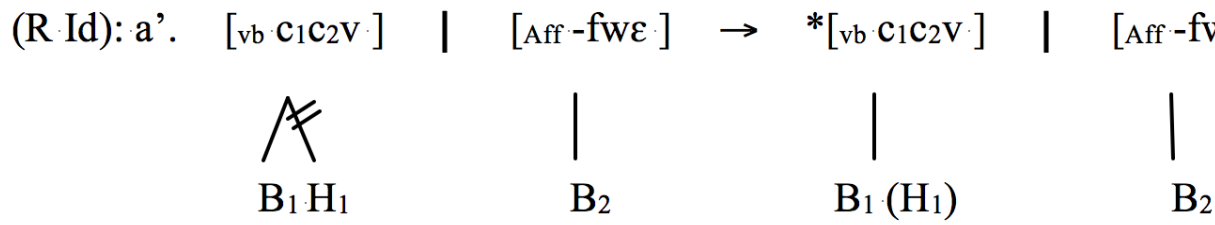

La condition est remplie pour que (RII) s'applique puisque $(H) \neq B$, au niveau II. 


\section{Niveau II}

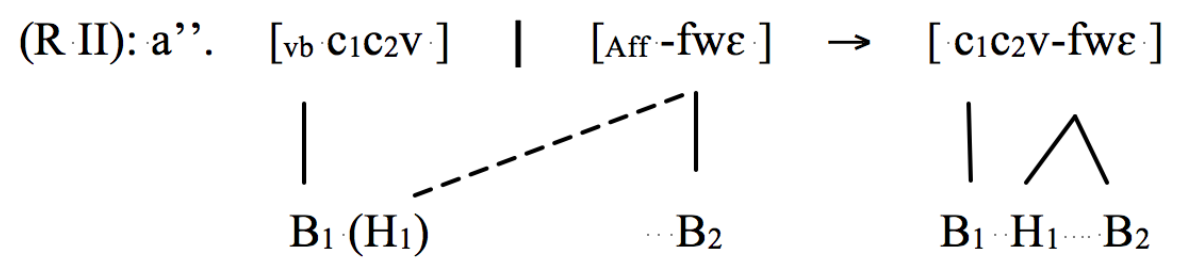

La forme attendue est donc obtenue au terme de l'application de (R II). Quel traitement donner à (23b) et (23c) ?

Niveau I

Pour la dérivation de (23b) et (23c), l'adjonction des suffixes au niveau I n'entraîne aucun effet dépresseur sur le ton des verbes. Il en résulte donc deux domaines morphophonologiques. Mais les structures obtenues au terme du processus, constituent les structures de base auxquelles peut s'appliquer la règle (R I'), ainsi que le montre (25) cidessous qui résume les nouveaux processus, les résultats étant les mêmes pour les suffixes -fwê (Agb, Cent) et $-l \hat{\varepsilon}$ (Agb, Gb, Cent), que nous renommons Suff:

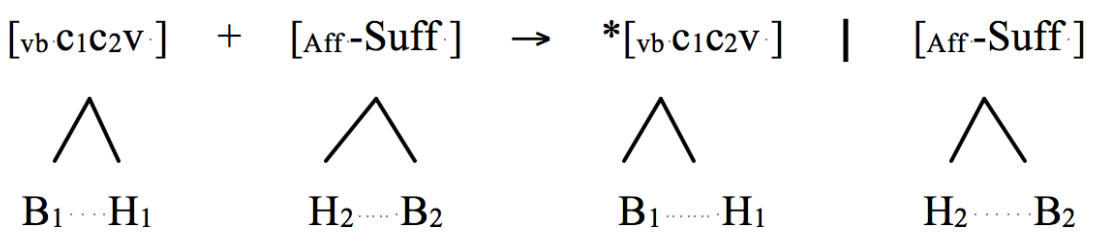

L'application de (R Id) donne ce qui suit en (26) :

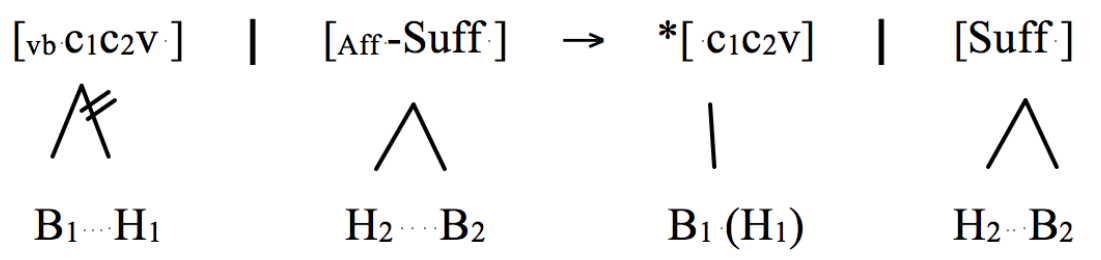

54 Au terme de la désassociation de $T_{2}$ verbal, nous obtenons une structure dans laquelle nous avons un ton haut flottant. Suit alors l'application de la règle (R Ie) pour effacer le Ton Haut flottant pour dériver les formes escomptées en (27) :

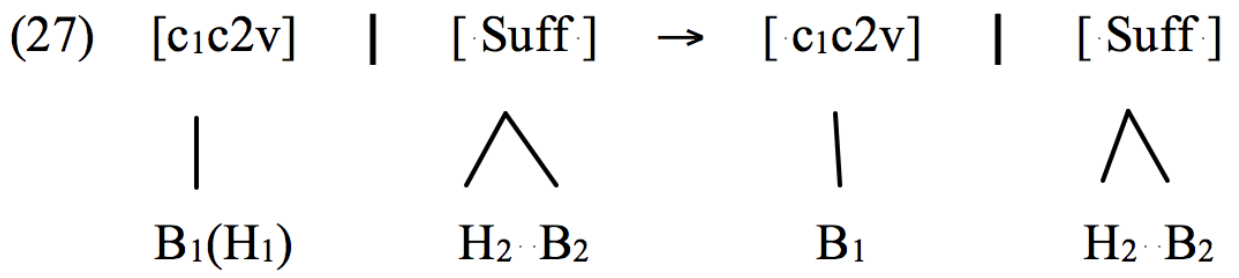

Comme on peut le constater, tout le processus de dérivation des formes ci-dessus s'est déroulé au niveau I puisqu'aucun processus tonal orienté vers le suffixe n'a été déclenché. - Avec les verbes de structure cvvv́ : 
Les mêmes faits relevés ci-dessus peuvent s'observer ici aussi mais cette fois les faits diffèrent de ceux qui précèdent, vu qu'un processus segmental de resyllabification doit intervenir nécessairement au niveau I.

\section{$\underline{\text { Niveau I }}$}

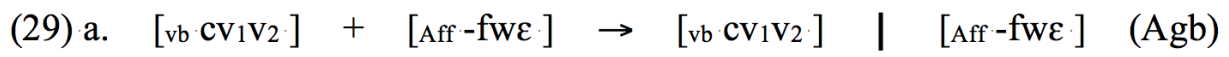

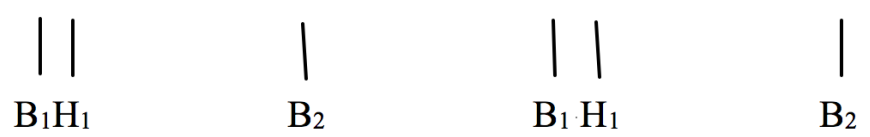

b. $\left[\mathrm{vb}^{\left.-\mathrm{CV}_{1} \mathrm{~V}_{2}\right]}+{ }_{\text {Aff }}-\right.$ Suff $\left.] \rightarrow{ }^{\mathrm{vb}} \mathrm{Cv}_{1} \mathrm{~V}_{2}\right] \mid$ [Aff - Suff $] \quad(\mathrm{Gb}$, Cent $)$

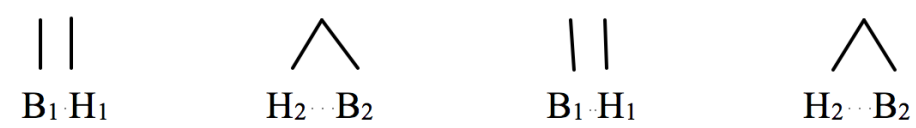

Au niveau I, l'adjonction des affixes n'entraîne aucun effet abaisseur sur le ton du verbe. Mais elle déclenche un processus de resyllabification dont le but est de faire passer le verbe de la structure cvv́ à la structure cč̌, le ton fondamental de cette dernière devant être réalisé Bas, ainsi que le montrent les analyses en (24)-(27) pour les exemples en (23) ci-dessus. Chaque étape de ce processus est franchie après l'application d'une règle.

(R I) Resyllabification

(R Ia) Règle de propagation

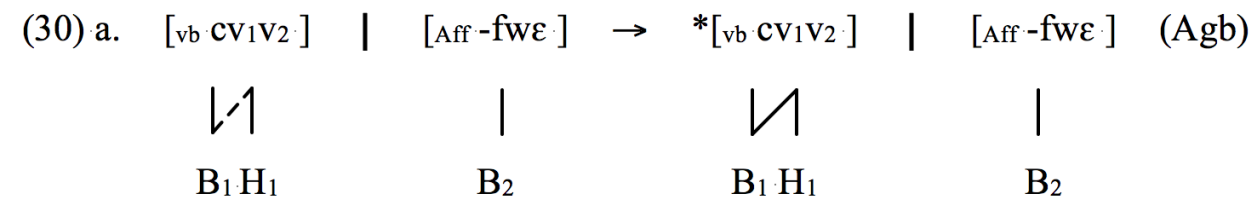

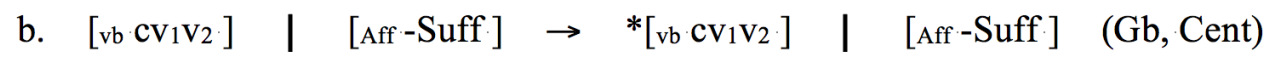

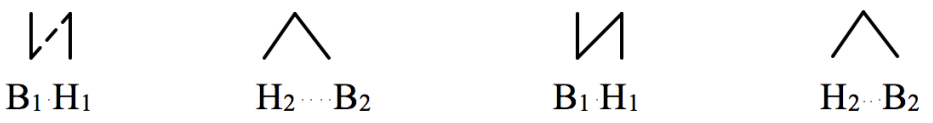

Les formes obtenues au terme de (30), ne sont pas celles attendues en (28). Elles se prêtent par conséquent à l'application de (R Ib). 
(31) a. $\quad\left[\mathrm{vb} \cdot \mathrm{c}_{1} \mathrm{~V}_{1} \mathrm{~V}_{2}\right] \quad\left|\quad[\mathrm{Aff}-\mathrm{fw} \varepsilon] \rightarrow{ }^{*}\left[\mathrm{vb}^{-} \mathrm{c}_{1} \mathrm{~V}_{1} \mathrm{~V}_{2}\right] \quad\right| \quad[\mathrm{Aff}-\mathrm{fw} \varepsilon] \quad(\mathrm{Agb})$

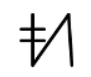

$\mathrm{B}_{1} \cdots \mathrm{H}_{1}$

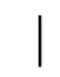

$\mathrm{B}_{2}$

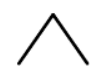

$\mathrm{B}_{1} \cdots \mathrm{H}_{1}$

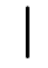

$\mathrm{B}_{2}$

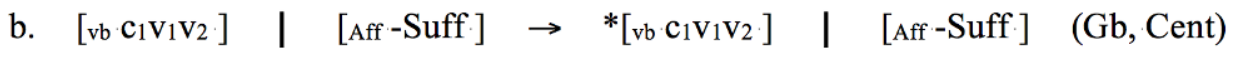
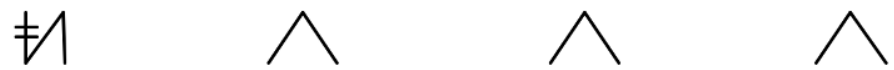
$\mathrm{B}_{1} \cdot \mathrm{H}_{1}$
$\mathrm{H}_{2} \cdots \mathrm{B}_{2}$
$\mathrm{B}_{1} \cdots \mathrm{H}_{1}$
$\mathrm{H}_{2} \cdots \mathrm{B}_{2}$

Dans les formes obtenues, $\mathrm{v}_{1}$ est une voyelle antérieure haute dépourvue de ton. Elle peut donc se palataliser comme le prévoit (R Ic) :

$(32)$ a
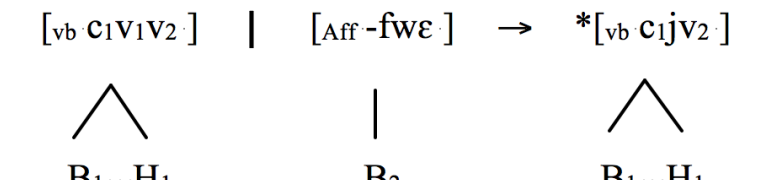

[Aff- $-\mathrm{fw} \varepsilon$ ]

(Agb)

$\mathrm{B}_{1} \cdots \mathrm{H}_{1}$

$\mathrm{B}_{2}$

$\mathrm{B}_{1} \cdots \mathrm{H}_{1}$

$\mathrm{B}_{2}$

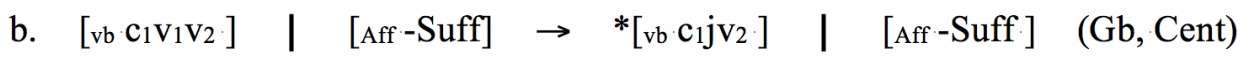

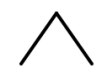

$\mathrm{B}_{1} \cdot \mathrm{H}_{1}$

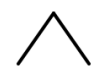

$\mathrm{H}_{2} \cdots \mathrm{B}_{2}$

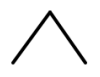

$\mathrm{B}_{1} \cdots \mathrm{H}_{1}$

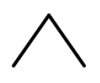

$\mathrm{H}_{2} \cdots \mathrm{B}_{2}$

61

$\mathrm{Au}$ terme de l'application de (R Ic), nous obtenons des formes qui ne sont pas celles escomptées et il y a une violation de l'OCP (Leben 1973), deux tons hauts $\mathrm{H}_{1}$ et $\mathrm{H}_{2}$ étant adjacents. La règle ( $R \mathrm{Id}$ ) va donc s'appliquer pour désassocier $T_{2}$, c'est-à-dire $H_{1}$ qui devient alors flottant.

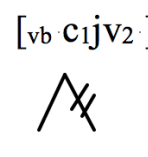

$\mathrm{B}_{1} \cdot \mathrm{H}_{1}$

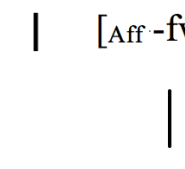

$\mathrm{B}_{2}$

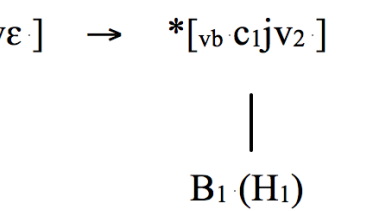

$[$ Aff - fwe $]$

$(\mathrm{Agb})$

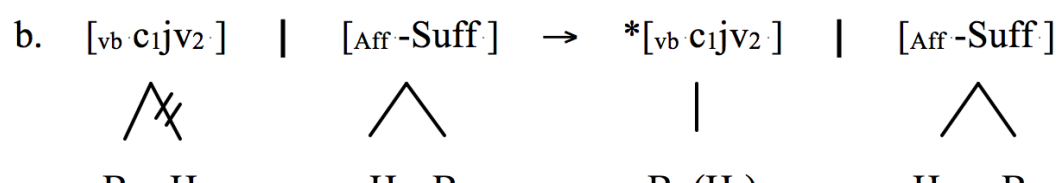
$\mathrm{B}_{1} \cdots \mathrm{H}_{1}$
$\mathrm{H}_{2} \cdots \mathrm{B}_{2}$
$\mathrm{B}_{1}\left(\mathrm{H}_{1}\right)$
$\mathrm{H}_{2} \cdots \mathrm{B}_{2}$

A ce niveau de l'analyse, la forme obtenue en (33b) sera soumise à la règle d'effacement du ton flottant (R Ie) afin d'obtenir la forme escomptée, avec la formation de deux

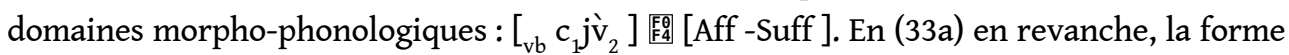
obtenue est soumise au facteur dialectal (en agba) qui va permettre de mettre en application, au niveau II, la règle (R II) dont l'effet est de propager le ton flottant issu du verbe vers le suffixe -fwè. Nous obtenons donc la forme escomptée. De cette règle résulte la formation d'un seul domaine morpho-phonologique, au niveau II : $\left.{ }_{\mathrm{vb}} \mathrm{c}_{1} \mathrm{j} \mathrm{v}_{2}-\mathrm{fw} \hat{\varepsilon}\right]$.

Les préfixes faibles, éléments préfixaux de circonfixes faibles, ne déclenchent aucun processus tonal. Ils forment donc un unique domaine morpho-phonologique indépendamment des autres morphèmes avec lesquels ils se combinent. $\grave{N}$-, comme 
indiqué plus haut, déclenche un processus segemental qui ne concerne pas directement notre analyse et qui pourra être traité dans un autre cadre que celui-ci.

L'affixe fort se manifeste par son effet d'abaissement du ton démarcatif Haut du verbe, au niveau I. Cet effet se traduit par l'absence de frontière morpho-phonologique entre l'affixe et le verbe mais aussi par l'abaissement automatique jusqu'au niveau Bas du ton démarcatif. Mais, au terme du processus pour le préfixe fort, il y a formation d'un ton modulé montant en position finale. Nous pouvons en rendre compte, en postulant que l'action du préfixe fort consiste à propager son ton Bas sur la position du ton démarcatif du verbe, simplement par l'application de la règle (R Ia). Avec les circonfixes, c'est plutôt l'élément suffixal fort qui entre en jeu. On peut admettre que dans le circonfixe, l'élément suffixal est hiérarchiquement plus fort que l'élément préfixal.

Notons que ces règles s'appliquent aux verbes de toute structure, à l'exception des verbes de structure ccv̌, qui portent déjà un ton modulé auxquels on n'aurait qu'à appliquer les règles $(R I d)$ qui désassocie et $(R$ Ie) qui efface le ton démarcatif Haut respectivement. Illustrons nos analyses avec àf̌̌ « fatigue » dérivé de f́́ « se fatiguer » et klèwâ « écriture ", à partir de $k l \underline{\varepsilon}$ « écrire ».

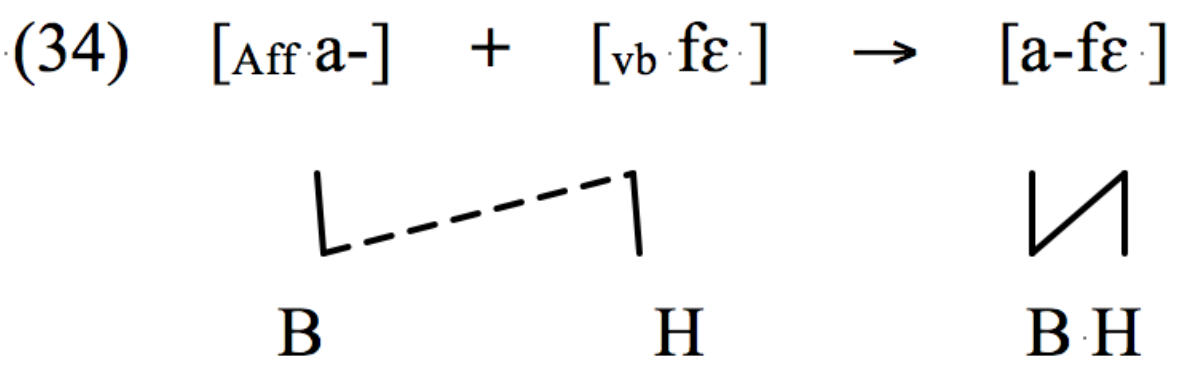

$(35)$
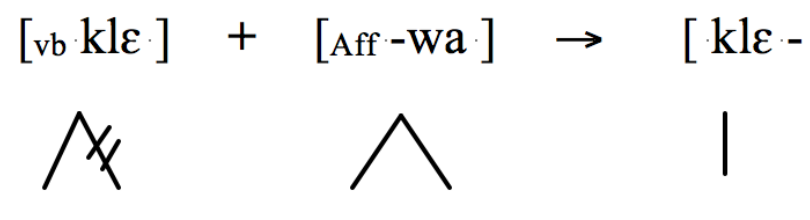

wa]

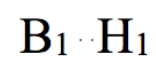

$\mathrm{H}_{2} \cdots \mathrm{B}_{2}$
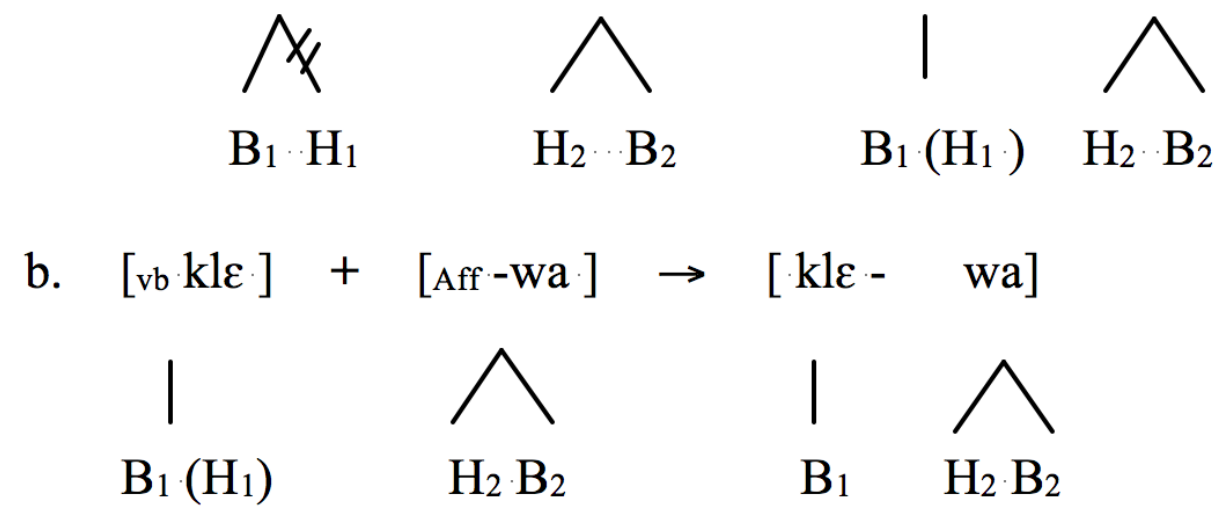

On peut attester du caractère simple et économique du cadre utilisé pour rendre compte des faits du baoulé. Il est simple car les faits tonals sont analysés en termes d'affixe forts 
ou faibles et de niveau, selon que les affixes déclenchent ou non un processus tonal et la direction dans lequel ce processus se fait. Il est économique car plusieurs faits peuvent être expliqués au moyen d'un nombre limité de règles. Qu'en est-il des faits de l'agni ?

\section{b. Le cas de l'agni}

Majoritairement, les analyses proposés pour expliquer les faits du baoulé peuvent être aussi évoquées pour l'agni. On peut voir en effet qu'en agni, les suffixes ou éléments suffixaux forts ont un effet systématiquement dépresseur sur le ton démarcatif Haut du verbe, au niveau I, dans les mêmes conditions qu'en baoulé. Cependant, les faits de l'agni ne permettent pas de dire qu'un quelconque processus tonal est orienté vers un affixe à partir du verbe. Tous les processus se déroulent au niveau I. A ce même niveau, se déroule le processus de resyllabification qu'impliquent les formes dérivées $\grave{s} s \bar{\imath} \grave{\varepsilon}$ " fait de rire " (de sìrí « rire »), èsjề « fait de diriger » (de sié « diriger, garder »), દ̀tjă « fait de crier » (de tiáa «crier »). Ces formes sont obtenues par l'application des règles (R Ia)-(R Id) évoquées plus haut. (R Ie) ne peut s'appliquer puisque $T$ flottant $\neq T$ suivant. La (R II) non plus ne peut s'appliquer car le processus ne se déroule qu'au niveau I. Le ton Bas flottant est donc présent et crée un effet downstep au contact du ton Haut qui suit et que nous notons ici par le ton Moyen. La représentation est èsjè donc la suivante :

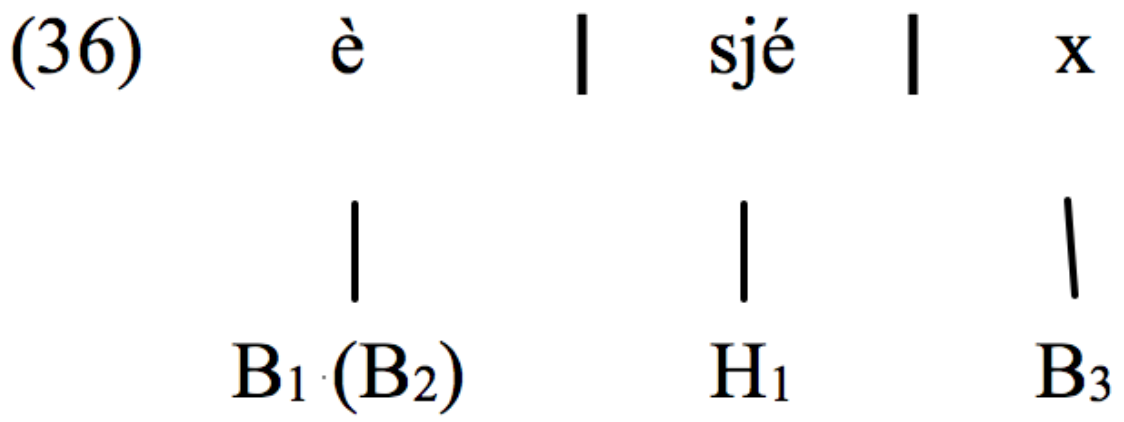

69 L'élément $\mathrm{x}$ dans le schéma symbolise de façon présumée l'élément suffixal à ton Bas d'un circonfixe. Sa présence est postulée en raison des faits observés en agni sanvi et en indénié (cf. ex. (12), plus haut). Dans ces parlers, en effet, on peut voir dans les formes dérivées un élément suffixal à ton Bas qui se manifeste sous la forme $-l \grave{\varepsilon}$ ou $-\grave{\varepsilon}$ ou encore comme un ton Bas quand il est explicité. C'est vraisemblablement cet élément que nous avons dans la forme $\grave{c} t a \grave{~}$ « fait d'être nourrice » en (16) plus haut. C'est aussi lui que nous avons dans les formes à nasale préfixale ìzérê « prière " en indénié et en ànó, gbàzâa " promenade ", ṅziê « don fait lors des funérailles », en indénié, et sous la forme -lì dans la forme ǹnàfil citées, la forme de base de l'élément suffixal est -lغ̀.

\section{Conclusion}

Nous avons démontré à travers cet article, l'existence, du point de vue fonctionnel, de deux classes d'affixes dérivationnels qui s'adjoignent aux verbes pour dériver des noms de procès, d'agent, de sens " possibilité de » et de lieux. Les affixes d'une classe, c'est-àdire les affixes forts ont un effet abaisseur sur le ton fondamental du verbe, tandis que ceux de l'autre classe, c'est-à-dire les affixes faibles n'ont pas cette propriété. Cependant, 
du fait des facteurs dialectal et contextuel, certains affixes du baoulé fonctionnent comme des affixes forts. On note également que pour les deux langues, à un premier niveau, le processus tonal en jeu est orienté de l'affixe fort vers le verbe, tandis qu'au deuxième niveau, en baoulé, le processus tonal est orienté du verbe vers le suffixe faible. Il en découle que lorsque l'adjonction d'un affixe ne déclenche pas de processus tonal, il y a formation de deux domaines morpho-phonologiques distincts [verbe] [Affixe]. En revanche, lorsqu'un processus tonal est déclenché, il y a formation d'un unique domaine morpho-phonologique [Verbe- Affixe].

71 Ce travail ne peut prétendre avoir épuisé toutes les questions relatives à l'adjonction de dérivatifs aux verbes dans les deux langues. Il y a par exemple la question du statut fort ou faible du préfixe $\grave{E}$ - en agni. Certains faits suggèrent de le considérer tantôt comme un

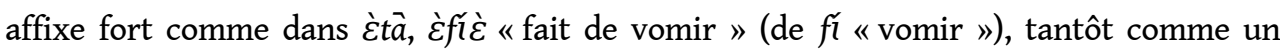

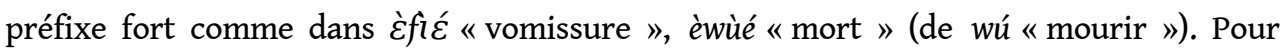
l'heure l'hypothèse la plus plausible est de considérer qu'il y a deux préfixes $\grave{E}-$.

\section{BIBLIOGRAPHIE}

Blench R. \& Kay W. (2000), « Niger-Congo », in B. Heine \& D. Nurse (eds.), African Languages : An Introduction, Cambridge, New-York, Melbourne, Madrid : Cambridge University Press, 347p.

Creissels D. (1995), Eléments de syntaxe générale, Paris : Presses Universitaires de France, 345p.

Creissels D. \& Kouadio N. J. (2010), « Ditransitive construction in baule », in Comrie B., Haspelmath M. \& Malchukov A. (eds.), Studies in Ditransitive Constructions : A comparative Handbook, Berlin : De Gruyter Mouton, pp. 165-189.

Creissels D. \& Kouadio N. J. (1977), Description phonologique et grammaticale d'un parler baoulé, Abidjan : ILA, 642p.

Diki-Kidiri M. (1977), Le Sango s'écrit aussi ... : esquisse linguistique du sango, langue nationale de l'empire centrafricain, Paris : SELAF, 187p.

Diki-Kidiri M. (2008), Le vocabulaire scientifique dans les langues africaines : Pour une approche culturelle de la terminologie, Paris : Karthala, 229p.

Guilbert, L. (1975), La créativité lexicale, Paris : Larousse, 286p.

Katamba F. (1993), Morphology, London : Palgrave MacMillan, 354p.

Keïta M. (2008), Système morpho-phonologique de l'agni : complexité vocalique, complexité tonale récupération du gabarit en agni, Université Denis Diderot, Paris 7, 380p.

Leben W. (1983), « Tone in the Verbal System of Anyi », in Proceedings of the 11th Conference on Africa Linguistics, Dordrecht : Foris, pp. 177-184.

Lilly R. \& Viel M. (1998), La prononciation de l'anglais : Règles phonologiques et exercices de transcription , Paris : Hachette Supérieur, 160p.

Matthew P. H. (1991), Morphology, Second Edition, Cambridge : Cambridge University Press, 251p. 
Nwankwegu J. A. (2013), Fundamentals of Morphology, Ebonyi State University, Nigeria : Envoy Media Group, 126p.

Sow A. I. (1966), « Remarques sur les infixes de derivation dans le fulfuldé du Fouta-Djalon », in Journal of West African Languages 3, 1, pp. 13-21.

Stewart J. (1989), « Kwa », in Bendor-Samuel (ed.), The Niger-Congo Languages, University Press of America, Lanham, pp. 217-247.

Welmers E. Wm. (1973), African Language Structures, Berkeley, Los Angeles, London : University of California Press, 488p.

\section{NOTES}

1. C'est ici pour nous l'occasion d'exprimer toute notre gratitude à nos informateurs qui sont Mme Sabine Nyamien Epse Chabbert, locutrice du parler central (Toumodi), Mlle Naomie Assiéoussou, étudiante de niveau Master en sciences du Langage, pour le parler agba (Bocanda) et M. Kouassi Johnson Nzué, également étudiant de niveau Master en Sciences du Langage, pour le parler gblo (Diabo).

2. Nous exprimons nos remerciements à Dr Ange Adou, pour les données des parlers ànó et indénié, et à Mlle Tamala Ahaté, Doctorante en Sciences du Langage.

3. «qui s'associe automatiquement à sa voyelle finale quand il (le verbe) n'est pas suivi d'un complément ou d'un adjoint »

4. La forme ìnàfílè est dérivée de dàfí « dormir ». Conformément à une règle phonologique, la consonne sonore / $d /$ se nasalise au contact du préfixe nasal homorganique.

\section{RÉSUMÉS}

L'article propose un traitement unifié des comportements tonals impliqués dans la dérivation de noms par adjonction d'affixes à des bases verbales en agni et en baoulé, deux langues kwa de Côte d'Ivoire. L'hypothèse qui sous-tend cette étude est que les affixes dérivationnels dans les langues étudiées et peut-être dans les langues du groupe Tano Central de la famille kwa se répartissent en deux groupes : les affixes faibles, qui n'ont aucun effet dépressif sur le schème tonal du verbe, et les affixes forts, caractérisés par leur effet dépressif sur le schème tonal du verbe. Selon la nature fonctionnelle des affixes, les schèmes segmental et tonal des bases verbales, un processus tonal est déclenché dans la direction affixe-verbe, à un premier niveau, et éventuellement, dans la direction verbe-affixe, à un deuxième niveau. Dans cette étude qui s'inscrit dans le cadre théorique de l'interface morphologie-phonologie nous abordons dans un premier temps les généralités sur le système tonal du verbe en baoulé et en agni, ensuite nous exposons les données recueillies dans des dialectes des deux langues et enfin, nous procédons à l'analyse et à l'interprétation des faits.

This article seeks to provide a unified account of different tonal behaviors when affixes combine with verb stems to derive nouns in anyi and baule, two languages of the kwa branch of Côte d'Ivoire. The underlying hypothesis in this study is that derivational affixes in the languages 
under study and perhaps in all languages of the Central Tano group of the kwa branch are subdivided into two groups: weak affixes, which have no depressive action on verb tone, and strong affixes, which have a depressive action on verb tone. Depending on the functional nature of affixes, the segmental and tonal structures of verb stems, tonal behavior in verb-affix combinations is verb-oriented from the affix, on a first level, and may eventually be suffixoriented, from the verb, on a second level. Carried out within the theoretical framework of the morphology-phonology interface, the study first deals with general overviews about the verbal tonal system in anyi and baule, then exposes data drawn from anyi and baule dialects and finally gives an account of the facts observed.

INDEX

Mots-clés : affixes dérivationnels, formes verbales, affixes forts, affixes faibles, domaine morpho-phonologique

Keywords : derivational affixes, verbal forms, strong affixes, weak affixes, morpho-phonological domain

\section{AUTEUR}

\section{ALAIN ALBERT ADEKPATÉ}

Université Félix Houphouët-Boigny, Abidjan, Côte d'Ivoire

alainadekpate@gmail.com 